\title{
PACKING COLORING OF SOME UNDIRECTED AND ORIENTED CORONAE GRAPHS
}

\author{
DaOuya Lä̈́he, Isma Bouchemakh \\ Faculty of Mathematics, Laboratory L'IFORCE \\ University of Sciences and Technology Houari Boumediene (USTHB) \\ B.P. 32 El-Alia, Bab-Ezzouar, 16111 Algiers, Algeria \\ AND \\ ÉRIC SOPENA \\ University Bordeaux, LaBRI, UMR5800, F-33400 Talence, France \\ CNRS, LaBRI, UMR5800, F-33400 Talence, France \\ e-mail: Eric.Sopena@labri.fr
}

\begin{abstract}
The packing chromatic number $\chi_{\rho}(G)$ of a graph $G$ is the smallest integer $k$ such that its set of vertices $V(G)$ can be partitioned into $k$ disjoint subsets $V_{1}, \ldots, V_{k}$, in such a way that every two distinct vertices in $V_{i}$ are at distance greater than $i$ in $G$ for every $i, 1 \leq i \leq k$. For a given integer $p \geq 1$, the $p$-corona of a graph $G$ is the graph obtained from $G$ by adding $p$ degree-one neighbors to every vertex of $G$. In this paper, we determine the packing chromatic number of $p$-coronae of paths and cycles for every $p \geq 1$.

Moreover, by considering digraphs and the (weak) directed distance between vertices, we get a natural extension of the notion of packing coloring to digraphs. We then determine the packing chromatic number of orientations of $p$-coronae of paths and cycles.
\end{abstract}

Keywords: packing coloring, packing chromatic number, corona graph, path, cycle.

2010 Mathematics Subject Classification: 05C15, 05C70, $05 \mathrm{C} 05$.

\section{REFERENCES}

[1] G. Argiroffo, G. Nasini and P. Torres, Polynomial instances of the packing coloring problem, Electron. Notes Discrete Math. 37 (2011) 363-368.

doi:10.1016/j.endm.2011.05.062 
[2] G. Argiroffo, G. Nasini and P. Torres, The packing coloring problem for $(q, q-4)$ graphs, Lecture Notes in Comput. Sci. 7422 (2012) 309-319. doi:10.1007/978-3-642-32147-4_28

[3] G. Argiroffo, G. Nasini and P. Torres, The packing coloring problem for lobsters and partner limited graphs, Discrete Appl. Math. 164 (2014) 373-382. doi:10.1016/j.dam.2012.08.008

[4] B. Brešar, S. Klavžar and D.F. Rall, On the packing chromatic number of Cartesian products, hexagonal lattice, and trees, Discrete Appl. Math. 155 (2007) 2303-2311. doi:10.1016/j.dam.2007.06.008

[5] J. Ekstein, J. Fiala, P. Holub and B. Lidický, The packing chromatic number of the square lattice is at least 12, March 12, 2010.

arXiv:1003.2291v1 [cs.DM].

[6] J. Ekstein, P. Holub and B. Lidický, Packing chromatic number of distance graphs, Discrete Appl. Math. 160 (2012) 518-524.

doi:10.1016/j.dam.2011.11.022

[7] J. Ekstein, P. Holub and O. Togni, The packing coloring of distance graphs $D(k, t)$, Discrete Appl. Math. 167 (2014) 100-106.

doi:10.1016/j.dam.2013.10.036

[8] J. Fiala and P.A. Golovach, Complexity of the packing coloring problem for trees, Discrete Appl. Math. 158 (2010) 771-778.

doi:10.1016/j.dam.2008.09.001

[9] J. Fiala, S. Klavžar and B. Lidický, The packing chromatic number of infinite product graphs, European J. Combin. 30 (2009) 1101-1113. doi:10.1016/j.ejc.2008.09.014

[10] A.S. Finbow and D.F. Rall, On the packing chromatic number of some lattices, Discrete Appl. Math. 158 (2010) 1224-1228.

doi:10.1016/j.dam.2009.06.001

[11] N. Gastineau, Dichotomies properties on computational complexity of S-packing coloring problems, Discrete Math. 338 (2015) 1029-1041. doi:10.1016/j.disc.2015.01.028

[12] W. Goddard, S.M. Hedetniemi, S.T. Hedetniemi, J.M. Harris and D.F. Rall, Broadcast chromatic numbers of graphs, The 16th Cumberland Conference on Combinatorics, Graph Theory, and Computing (2003).

[13] W. Goddard, S.M. Hedetniemi, S.T. Hedetniemi, J.M. Harris and D.F. Rall, Broadcast chromatic numbers of graphs, Ars Combin. 86 (2008) 33-49.

[14] D. Korže and A. Vesel, On the packing chromatic number of square and hexagonal lattice, Ars Math. Contemp. 7 (2014) 13-22.

[15] D. Laïche, Sur les nombres broadcast chromatiques, Magister Thesis, University of Sciences and Technology Houari Boumediene, Algeria, 2010 (in French). 
[16] D.F. Rall, B. Brešar, A.S. Finbow and S. Klavžar, On the packing chromatic number of trees, Cartesian products and some infinite graphs, Electron. Notes Discrete Math. 30 (2008) 57-61.

[17] C. Sloper, An eccentric coloring of trees, Australas. J. Combin. 29 (2004) 309-321.

[18] R. Soukal and P. Holub, A note on the packing chromatic number of the square lattice, Electron. J. Combin. 17 (2010).

[19] O. Togni, On packing colorings of distance graphs, Discrete Appl. Math. 167 (2014) 280-289.

doi:10.1016/j.dam.2013.10.026

[20] P. Torres and M. Valencia-Pabon, The packing chromatic number of hypercubes, Discrete Appl. Math. 190-191 (2015) 127-140.

doi:10.1016/j.dam.2015.04.006

[21] A. William, I. Rajasingh and S. Roy, Packing chromatic number of enhanced hypercubes, Internat. J. Math. Appl. 2 (2014) 1-6.

[22] A. William and S. Roy, Packing chromatic number of certain graphs, Internat. J. Pure Appl. Math. 87 (2013) 731-739.

doi:10.12732/ijpam.v87i6.1

[23] A. William, S. Roy and I. Rajasingh, Packing chromatic number of cycle related graphs, Internat. J. Math. Soft Comput. 4 (2014) 27-33.

Received 18 March 2016

Revised 6 June 2016

Accepted 6 June 2016 